\title{
Risk and risk prediction error signals in anterior insula
}

\author{
Peter Bossaerts
}

Received: 1 December 2009/Accepted: 21 April 2010/Published online: 29 May 2010

(C) Springer-Verlag 2010

\begin{abstract}
Most accounts of the function of anterior insula in the human brain refer to concepts that are difficult to formalize, such as feelings and awareness. The discovery of signals that reflect risk assessment and risk learning, however, opens the door to formal analysis. Hitherto, activations have been correlated with objective versions of risk and risk prediction error, but subjective versions (influenced by pessimism/optimism or risk aversion/tolerance) exist. Activation in closely related cortical structures has been found to be both objective (anterior cingulate cortex) and subjective (inferior frontal gyrus). For this quantitative analysis of uncertainty-induced neuronal activation to further understanding of insula's role in feelings and awareness, however, formalization and documentation of the relation between uncertainty and feelings/awareness will be needed. One obvious starting point is the link with failure anxiety and error awareness.
\end{abstract}

Keywords Anterior insula $\cdot$ Risk $\cdot$ Risk prediction error . Ambiguity · Uncertainty

\section{Introduction}

The anterior insula has been implicated in the translation of bodily state into feelings (Craig 2002) and perhaps even

\footnotetext{
P. Bossaerts $(\square)$

Computational and Neural Systems,

California Institute of Technology,

M/C 228-77, Pasadena, CA 91125, USA

e-mail: pbs@hss.caltech.edu

P. Bossaerts

Ecole Polytechnique Fédérale Lausanne,

Lausanne, Switzerland
}

into awareness (Craig 2009), and therefore, it plays a crucial role in the James-Lange theory that posits that emotional feelings are the result of physiological changes in one's body. Recent evidence (Preuschoff et al. 2008) suggests that, at the same time, the anterior insula is engaged in at least one, purely mathematical task, namely the tracking of risk. This involvement in rather cool calculation does not necessarily contradict the idea that anterior insula is also the gateway for subjective experience. Indeed, the Somatic Marker Hypothesis postulates, among others, that rational decision theory requires emotional anticipation of the outcomes (Bechara et al. 1997), and hence, that seemingly cool behavior and emotional decision making are intertwined.

Emotions, feelings and awareness are illusive concepts, generally defying formal analysis. Measurements are qualitative, or, at best, ordinal. This has hampered efforts to delineate precisely the contribution of anterior insula in the formation of awareness of one's (emotional) "self." The involvement of anterior insula in the encoding of precise quantitative features of the environment, namely, risk, may provide an opportunity to improve inference. Indeed, risk can be quantified in precise ways (Preuschoff and Bossaerts 2007; Preuschoff et al. 2008); formulae for its subjective counterpart exist (Christopoulos et al. 2009); and awareness can be tested using side-bets (Koch and Preuschoff 2007).

The purpose of this essay is (1) to clarify why risk assessment is important, (2) to introduce the reader to the measurement of risk and risk prediction error, (3) to elucidate the role of anterior insula in assessment of risk, and (4) to elaborate on the difference between objective and subjective risk and whether risk encoding in anterior insula is subjective. Many questions will remain unanswered, especially with respect to awareness of risk, for which the "Discussion" section elaborates. 


\section{Why is risk assessment important?}

This question may at first seem odd: most humans appear to be sensitive to risk, somehow they must be assessing risk. In classical decision theory, however, risk aversion (or its reference-dependent counterpart, loss aversion) emerges not as the result of an evaluation of risk, to be traded off against average expected rewards, but as a mere consequence of nonlinearity in the subjective utility of the reward. Specifically, risk aversion emerges when the incremental utility decreases as more reward is given; likewise, loss aversion is the consequence of higher incremental utilities for rewards in the loss domain than in the gain domain.

As a result, the decision maker may behave in a risk or loss averse way without having to keep track of risk. Nonlinear encoding of rewards would be sufficient to generate behavior that looks as if the decision maker is risk or loss averse (Koenig and Simmons 1994; Mihatsch and Neuneier 2002; Pratt 1964). The brain does not need to track risks independently of rewards.

One of the most convincing pieces of evidence in favor of nonlinear encoding of rewards came up recently in a human imaging study (Tom et al. 2007), where the usual striatal activation associated with reward prediction errors was found to exhibit the very nonlinearity that was consistent with the loss aversion revealed in subjects' choices. As a result, subjects' aversion to (downside) risk could have merely been attributed to (subjective) nonlinearity in the encoding of reward prediction errors. To generate the behavior, no separate encoding of risk was needed.

To be more precise (Tom et al. 2007) probed subjects' preferences for gambles in the gain and loss domain separately, and found that marginal utility for reducing losses was greater than marginal utility for increasing gains. As such, subjects exhibited loss aversion. This differential in marginal utility was reflected in striatal activation: deactivation for losses was higher per dollar than activation for gains.

While risk encoding, therefore, is not needed to explain risk-sensitive choices, it may be crucial for learning of rewards in a stochastic (i.e., uncertain) environment. Indeed, learning can be enhanced dramatically by modulating the learning rate depending on the risk. While this is explicit in Bayesian learning (e.g., the Kalman filter), the intuition is quite simple. When risk is high, one's prediction errors are likely to be high, so outcomes (rewards) are very noisy and one should not read much in one's prediction errors. As a result, the learning rate should be low. Conversely, if risk is low, prediction errors are significant, and the learning rate high.

Interestingly, reward-error-related firing of dopaminergic neurons in the non-human primate brain appears to reflect this: neuronal activation is higher when prediction risk is lower (Tobler et al. 2005). One should consider that to be evidence that risk is encoded in the brain, albeit indirectly. This finding of risk-adapted encoding of reward prediction errors has recently been confirmed in human imaging analysis (Bunzeck et al. 2010). Traces of direct risk encoding in dopaminergic neurons has been found in the nonhuman primate brain (Fiorillo et al. 2003) and in sub-cortical dopaminoceptive regions of the human brain (Preuschoff et al. 2006).

However, the relation between risk and learning rate is complex. When risk increases because the environment has changed, learning rates should increase, so that recent prediction errors are weighted more heavily, while prediction errors from the past (and before the change in the environment) are weighted less. Overall, when the environment changes regularly (is unstable), the learning rate should be higher. Plenty of behavioral support for this type of adaptation of the learning rate with the stability of the environment has emerged in recent years (Behrens et al. 2007; Nassar et al. 2009; Nursimulu et al. 2009). fMRI analysis points to a crucial role of the anterior cingulate cortex (ACC) in adaptation of learning rates (Behrens et al. 2007).

\section{How to measure risk?}

Risk has multiple facets. There is the typical size of one's prediction error (the variance, i.e., the expected squared deviation of the outcome from one's prediction). In addition, one may weigh differently negative prediction errors (skewness, i.e., the expected third-order power of the prediction error; or the expected shortfall, i.e., the expected prediction error provided it is negative). In addition, one may weigh outliers more heavily (kurtosis, i.e., the expected fourth-order power of the prediction error), etc.

Decision theory prescribes, however, that risk is to be measured foremost by variance. This is made explicit in mean-variance choice theory (Kroll et al. 1984) or Bayesian updating in a Gaussian world (Berger 1985), and implicit in standard central limit theorems (where the asymptotic distribution of predictions depends only on the variance; Schervisch 1997). Not that skewness and kurtosis are unimportant; their relevance is, however, only of second order.

One interesting aspect of variance as a metric of risk is its symmetry: positive prediction errors are weighted equally heavily as negative prediction errors. This is counter-intuitive in a choice context: one would expect negative prediction errors (losses) to be weighted more heavily. For learning, however, positive prediction errors are equally important as negative prediction errors when 
setting one's learning rate. Indeed, to ignore positive prediction errors would be to throw away crucial information about the risk in the environment needed to set one's learning rate optimally.

Proper assessment of risk pre-supposes that one has learned the risk. As with reward learning, the crucial ingredient to risk learning is the risk prediction error. This equals the outcome (realized risk) minus anticipation (expected risk). When measuring the anticipation as variance, it is the difference between the size (measured as the square) of the prediction error minus the expectation of this size (the expected squared prediction error, and hence, the variance). Proper updating of the risk requires encoding of this risk prediction error.

As we shall document later, activation in the anterior insula reflects both (anticipated) risk and a risk prediction error, where risk is measured as variance (of the reward prediction error), and the risk prediction error is simply the squared prediction error minus its expectation. As such, anterior insula is known to play a crucial role in tracking of risk.

\section{Encoding of risk and risk prediction errors in anterior insula}

Some early studies implicated anterior insula in risk tracking (Huettel et al. 2005; Kuhnen and Knutson 2005; Paulus et al. 2003), but risk was dealt with informally, even if quantitatively. In Huettel et al. (2005), the risk was measured in terms of probability of an odd event, with probability ranging from 0.20 to 0.50 . When measured as variance, risk does increase with this probability, but nonlinearly. In addition, task performance decreased with probability of the odd event, so that insula activation could be attributed to frustration rather than risk. Kuhnen and Knutson (2005) used the same metric of risk, and showed that anterior insula activation predicted risk avoiding choices even after accounting for their risk measure. In Paulus et al. (2003), risk "built" over time (risk-taking increased as subjects deliberately waited to respond), so insula activation may have reflected general attention, as in Brass and Haggard (2007).

In Preuschoff et al. (2006, 2008), risk was dissociated from expected reward to avoid confounding factors. The task was simple. Each trial, a randomly shuffled deck of 10 cards numbered 1-10 was presented. Two cards were going to be pulled out, consecutively. The subject first had to predict whether the second card was going to be higher or not. If she was right, she won $\$ 1$; otherwise she lost $\$ 1$. Once the bet was submitted, the first card was pulled out, and after a delay, the second card.

In this task, after seeing the first card, expected reward increases linearly with the chance of winning (conditional on the number on the card); risk (variance), however, first increases and then decreases, to produce an inverted-U pattern with minimum at the end points (zero and unit probability of winning) and maximum in the middle $(50 \%$ chance of winning) (see Fig. 1a).

Using fMRI, two types of activations were localized in the human brain, each with different timing duration. Specifically, in the delay period between presentation of the first card, phasic activations (those with a duration of $<1 \mathrm{~s}$, modulo the hemodynamic response delay) were searched for, as well as delayed activations (those that started $1 \mathrm{~s}$ or more after card presentation, and lasted until the end of the delay period, modulo the hemodynamic response) (see Fig. 1b). Within the set of phasic activations, those that changed linearly with (mean-adjusted) probability of reward were dissociated from those that changed nonlinearly with probability of reward. The latter were identified when the activation changed significantly as a function of the square of the mean-adjusted reward probability. Thus, four groups of activations were obtained.

Here, we are most interested in activations detected in anterior insula. Significant activations were found both phasic and delayed. The former increased with the square of the mean-adjusted reward probability, suggesting a Ushaped pattern as a function of reward probability, whereas the latter decreased, indicating that activation is an inverted-U function of reward probability (see Fig. 1c). Laterality is more pronounced for the delayed activation, with a much larger cluster size (and significance) in left anterior insula, but all patterns discussed below are the same in the right anterior insula. Importantly, there were no regions within anterior insula where activation changed linearly with the reward probability, neither phasic nor delayed. As such, all activations in anterior insula appear to roughly conform to the pattern in Fig. 1a, although the phasic activation has the opposite sign, that is, it is U-shaped as a function of reward probability, not inverted-U-shaped.

The voxel with peak activation was then retained and average activation across subjects was plotted against probability of reward, to verify that the pattern of activation is indeed quadratic, with extrema at $50 \%$ probability and at 0 and $100 \%$ probability. This was indeed the case for the delayed activation (see Fig. 2a). The activation exhibits the inverted-U-shaped pattern that is required for it to reflect encoding of risk (variance). The signal is delayed, which may be attributed to the fact that risk is forwardlooking: it concerns the uncertainty that is anticipated to be resolved when card 2 is shown. Because of its anticipatory nature, one can reasonably conjecture that the signal is time-locked to the outcome (when card 2 is displayed). Future studies could confirm this, by changing the delay to display of card 2 and verifying that peak activation moves accordingly. 
A

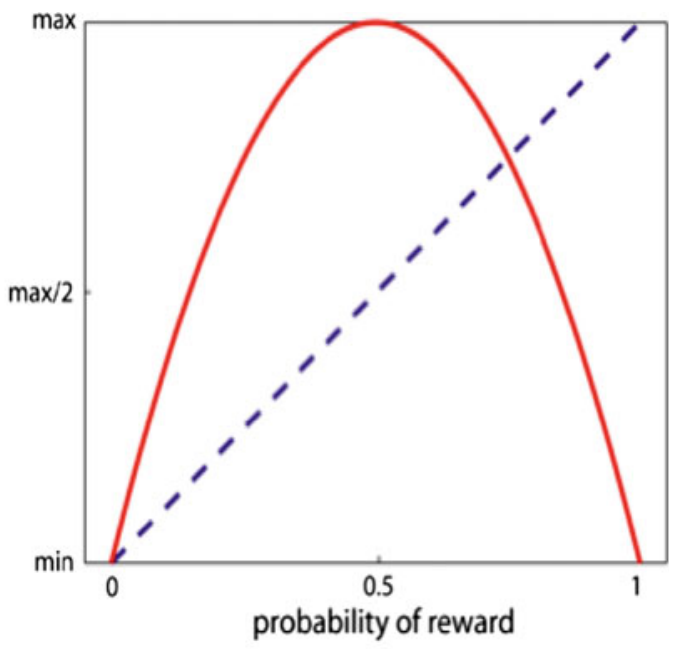

B

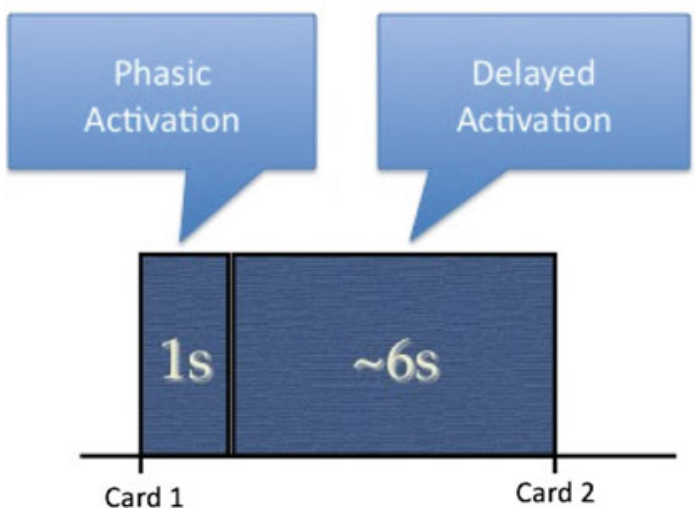

C

\begin{tabular}{|ccccccc|}
\hline Timing & Left/ Right & Mean & Mean & Mean & Cluster & $\begin{array}{c}\text { Max } t \\
\end{array}$ \\
& & $x$ & $y$ & $z$ & Size & Statistic \\
\hline Phasic & L & -31 & 14 & -2 & 41 & 7.3 \\
Phasic & $\mathrm{R}$ & 32 & 15 & -3 & 46 & 7.3 \\
Delayed & $\mathrm{L}$ & -31 & 22 & 8 & 82 & -8 \\
Delayed & $\mathrm{R}$ & 33 & 21 & 8 & 25 & -5 \\
\hline
\end{tabular}

Fig. 1 a Expected reward (dashed line) increases linearly in the reward probability; risk (red solid line), measured as reward variance, increases for reward probabilities below 0.5, then decreases; the relationships is quadratic, with equal minima at zero and unit probability; from Preuschoff et al. (2006). b Phasic activation refers to neuronal signals during the first second after display of the first card; delayed activation refers to neuronal signals during the remainder of the delay period between display of the first and second cards; from Preuschoff et al. (2006). c Table of location of BOLD signals in insula that change nonlinearly (quadratic relation) with reward probability. Sign of $t$ statistic indicates whether relationship is U-shaped (positive) or inverted-U (negative); from supplementary online material of Preuschoff et al. (2008), with signs added to indicate directionality. Only nonlinear changes of insula activation with reward probability emerged; the lack of linear correlation would suggest that insula does not encode expected reward

Identification of the nonlinear phasic activation in anterior insula turned out to be more tricky. It indicates correlation with the risk prediction error, that is, it reflected the difference between the actual size of the prediction error when card 1 was shown and the anticipated size. The prediction error we are referring to here is the difference between the actual reward forecast based on the number on card 1 and its expectation before display of card 1. Figure $2 b$ plots (in red) the average delayed activation, across subjects, stratified by level of risk prediction error. The relationship is linear and increasing.

The risk prediction error is backward-looking: it refers to the difference between an outcome (the size of the prediction error) and its anticipation before revelation (the prediction risk). Notice that there is also a risk prediction error upon display of card 2. It equals the difference between the actual magnitude of the reward-error before seeing card 2 and its expectation after seeing card 1 . Average activation in anterior insula across subjects is plotted in Fig. 2b in blue, for each of the various values that the risk prediction error could take on across trials. There is one outlier, namely, when the risk prediction error is zero. This happens only in trials where, after seeing the first card, there is no risk left anymore (the first card is either a 1 or a 10), so there is no risk prediction error either.

To contrast the timing of the activation related to anticipation and to error, Fig. 3a displays the time courses for trials that are stratified by level of risk and risk prediction error (left anterior insula). Differentiation of the anticipatory risk time courses builds only slowly, and peaks when risk is realized (display of card 2). In contrast, differentiation of the risk prediction error time courses follows immediately upon display of card 1 , and peaks at the usual hemodynamic response delay $(\sim 4 s)$. Figure $3 b$ localizes the risk and risk prediction error activations.

Activation in anterior insula correlating with risk prediction error has been independently verified in an fMRI analysis of the Iowa Gambling Task (d'Acremont et al. 2009b); there, risk prediction errors emerged after each draw from a deck. Risk prediction errors were measured as in Preuschoff et al. (2008).

\section{Objective and subjective risk}

In the aforementioned studies, risk (and risk prediction error) is objective. Magnitudes are computed from the actual chance numbers and outcomes, without any attempt to impute subjectivity, through risk aversion. Perceived risk could be magnified in risk averse agents, while differences in objective risks may become insignificant for risk-neutral agents. Evidence has recently emerged that risk encoding in ACC is objective, while activation in inferior frontal gyrus (IFG) correlating with risk strongly increases with risk aversion as revealed through subjects' choices (Christopoulos et al. 2009). 
Fig. 2 Axial cross-section (left panel) of the human brain with localization of delayed activation that decreased in the squared deviation of reward probability from 0.5 . Left and right anterior insula (ins) emerge, and, in the same (axial) plane, midbrain (thalamus) activation $(m d)$. Pattern of mean activation (right panel) (with standard errors) as a function of reward probability across 19 subjects in peak voxels of left and right anterior insula. Coronal cross-section (left panel) of the human brain with localization of phasic activation after card 1 that increased in the squared deviation of reward probability from 0.5 . Pattern of mean activation (right panel) (with standard errors) as a function of risk prediction error across 19 subjects in peak voxel of right anterior insula. Shown are mean activations after display of card 1 (red) as well as mean activations in the same voxel after display of card 2 (blue)
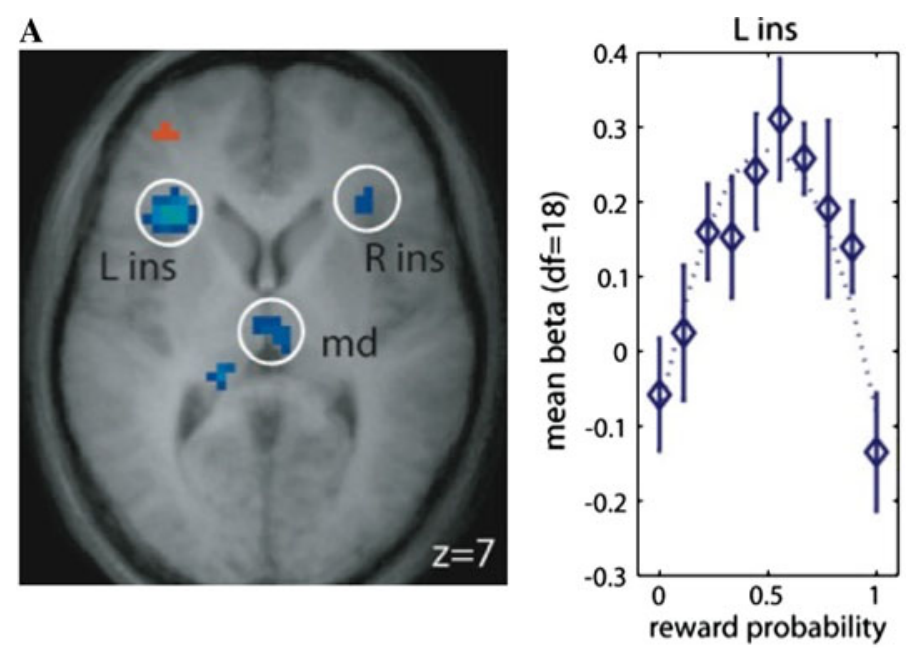

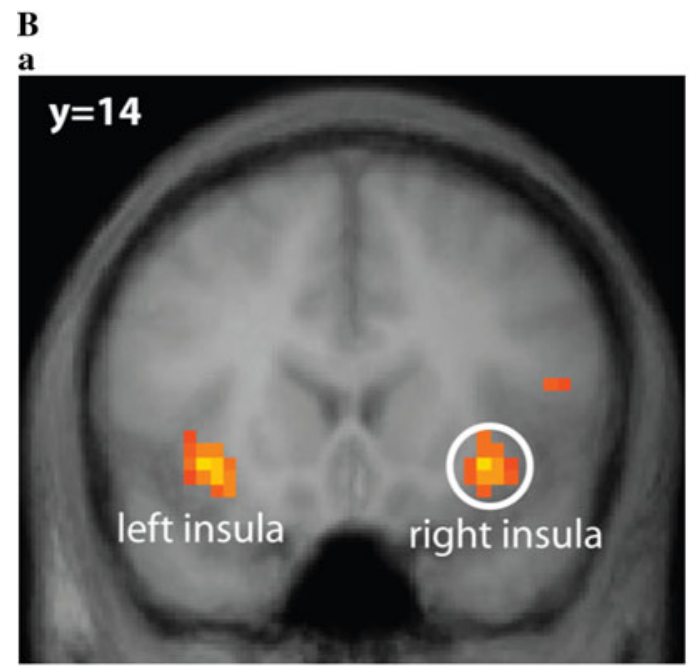

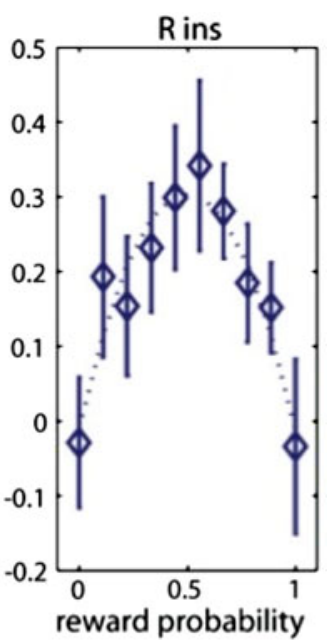

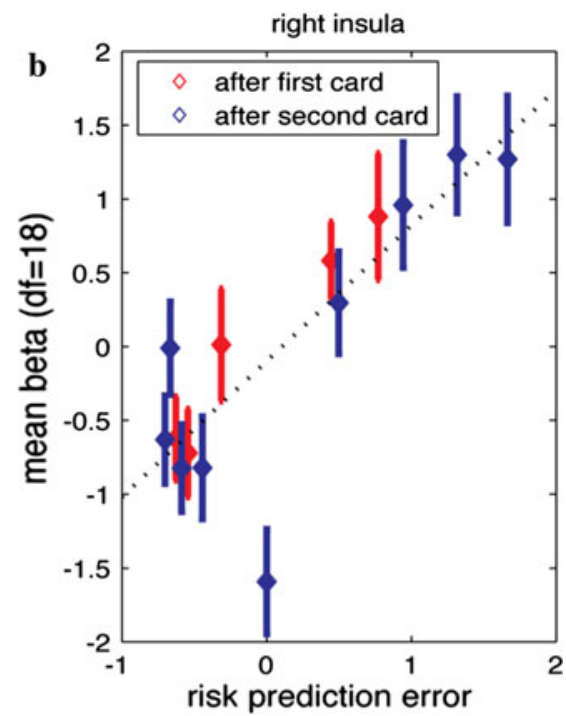

It is not known whether risk activation in anterior insula reflects subjective risk. The finding that risk activation in the closely connected IFG is subjective may carry over to the anterior insula, but the delay period between display of stimulus and outcome in (Christopoulos et al. 2009) included a choice epoch, which could have interfered with the delicate timing of risk and risk prediction error signal. While a (subjective) risk signal emerged (unpublished material), it did not survive whole brain correction. The conjecture is, however, that risk encoding in anterior insula is subjective, as anterior insula is thought to be crucial in the formation of subjective feelings (Craig 2009). The origin of all these risk-induced signals is not known. Common innervation and mutual connectivity would suggest that activations in insula, anterior cingulate and IFG are related.

Subjectivity can be measured in different ways than just risk aversion. Pessimism (overweighting of loss probabilities) is one of them. Pessimism features prominently in prospect theory, and recent imaging evidence shows that expected reward signals in striatal regions correlate with subjects' pessimism (Berns et al. 2008; Hsu et al. 2009). Further work is needed to elucidate the nature of subjectivism in stochastic reward environments.

\section{Discussion}

From its role in emotions, the involvement of insula in risk tracking should not come as a surprise. The lack of predictability of an event requires the body to be ready to take the right action in case the event materializes. Imagine, for instance, that a predator may be hiding somewhere with some probability. At a minimum, the heartbeat should increase, to be ready to react (fight/flight) in case the predator does appear. Preparedness should increase with uncertainty, and so should the heartbeat. The research on such cardiac defense response is vast (Ramirez et al. 2005), 
Fig. 3 Time courses of phasic (right) and delayed (left) BOLD signal in left anterior insula, stratified by level of prediction risk error (right) or level of risk (left); from Preuschoff et al. (2008). Sagittal cross-section of the human brain with localization of clusters of significant delayed activation that decreases in the squared probability of reward (reflecting risk; blue) and significant phasic activation that increases in the squared probability of reward (reflecting risk prediction error; yellow). Time courses in a are for peak voxels in these clusters
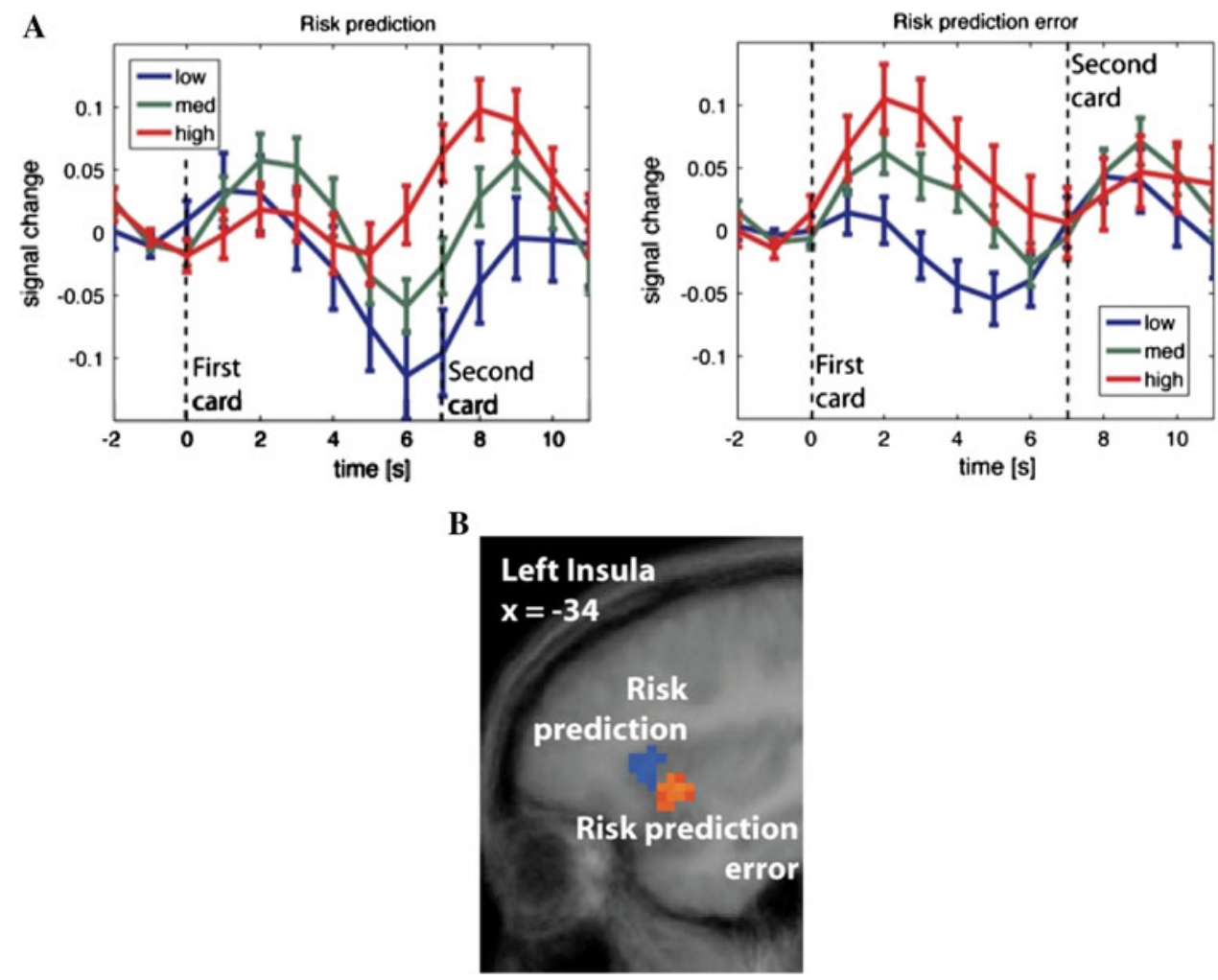

but association with uncertainty has yet to be fully explored.

The anterior insula has not only been implicated in awareness of one's own emotions, but in empathizing with those of others as well (Singer and Lamm 2009). To the extent that other people's emotions convey risk measurement, are one's own subjective risk assessments affected? If so, one can easily envisage social contagion of risk assessment [while the economics literature on contagion actually focuses on transmission of reward shocks rather than risk assessment (Bekaert et al. 2005)] [see Singer et al. (2009) for further discussion of the link between risk assessment and empathy].

Some of the evidence regarding the functionality of anterior insula can be re-interpreted in terms of risk assessment. For instance, the increase in activation following a low return in the trust game (Delgado et al. 2005) or a low offer in the ultimatum game (Sanfey et al. 2003) could be a risk prediction error-one did expect some variation in the opponent's actions, but the repayment or the offer is way beyond one's beliefs. The absence of insula activation in borderline personality disorder patients (KingCasas et al. 2008) can be seen as a lack of updating of prediction risk, which could explain their inability to recalibrate beliefs about opponents' strategies. Increases in insula activation upon mis-timing of otherwise well-known rhythms (Platel et al. 1997) likewise can be re-interpreted in terms of risk tracking: subjects should feel that they can predict familiar rhythms well, i.e., they anticipate small prediction errors, but a mis-timing reveals that prediction errors are larger than expected; in other words, a positive risk prediction error occurs, and insula signals this accordingly.

Anterior insula is by no means the only brain structure where risk and risk prediction errors are encoded. We already mentioned ACC and IFG before, which, while interconnected with anterior insula, play distinct roles [ACC encodes objective risk, while IFG risk signals correlate with risk aversion; (Christopoulos et al. 2009)]. Together with amygdala, anterior insula, ACC and IFG are thought to form a network of emotional salience (Seeley et al. 2007), so activation of risk in any of these structures should not come as a surprise.

The role of amygdala needs further elaboration, however. In pure risk tasks, where outcome probabilities are known or have been learned, like in the card game we discussed earlier, amygdala is rarely if ever activated. Amygdala emerges as a crucial brain region in contexts where outcome probabilities are not known (yet) (Hsu et al. 2005). Economists would refer to such situations as "ambiguous," or using language from statistics, the uncertainty involved could be called "estimation uncertainty." One can also observe this in single-unit recordings of neuronal firing in the amygdala-hippocampus complex 
(Rutishauser et al. 2006). Therefore, while insula is engaged in tracking of pure risk, amygdala seems to be functionally specialized in tracking estimation uncertainty.

To elaborate, "risk" is what remains after learning is finished; it is unavoidable, irreducible uncertainty (e.g., even the best weather forecasts are imperfect), and risk is encoded in anterior insula. In contrast, estimation uncertainty is the amount of uncertainty that can be reduced over time through learning (the novice weatherman can improve forecasts with experience). Amygdala signals the latter. To confirm this, future studies should attempt to correlate changes in estimation uncertainty (as measured, e.g., by Bayesian posterior variance) and activation in amygdala.

The old distinction of the functions of amygdala and insula as reflecting "fear" and "disgust," respectively, capture well the difference between ambiguity and pure risk. Most people fear the former [they are ambiguity averse, to the point that they make irrational decisions (Ellsberg 1961)], but they are not afraid of risk; they just wish it were not there.

While risk assessment and risk learning can be quantified precisely, and therefore, provide an opportunity to study the wider role of anterior insula in humans' ability to build subjective feelings from their bodily state and to become aware of them, the mathematics involved is more complicated than for, say, reward assessment and learning.

For instance, in reward prediction, the best forecast of one's intermediate forecast of a final outcome is the same as the best forecast of the final outcome. This is the consequence of a mathematical property of expectations, called the law of iterated expectations. Unfortunately, the law does not apply to risk when risk is measured as variance. As such, what one expects to be the typical mistake in predicting the final outcome is not the same as the anticipated magnitude of the change in one's intermediate forecasts. The mathematical complexity raises the question of how the human brain manages to cope with it [see d'Acremont et al. (2009a) for details].

We discussed the relevance of risk assessment for reward learning. In particular, optimal learning requires one to change the learning rate depending on the environment. How exactly the learning rate is to be changed is complicated (Preuschoff and Bossaerts 2007), which again makes one wonder how the brain may go about optimizing the learning rate. One suggestion was made recently (Bossaerts and Preuschoff 2009).

Most accounts of anterior insula focus on its role in translating emotions into feelings and awareness. These are intangible, elusive concepts. Yet at the same time, signals in anterior insula point to involvement in a very concrete and readily quantifiable task, namely, risk assessment. It is to be hoped that further exploration of this involvement would at the same time enhance our understanding of anterior insula's more difficult functionality.

For this to happen, however, the relationship between uncertainty and feelings/awareness will have to be studied more formally. Not much is known about the emotional impact of volatility. Lo and Repin (2002) provides emerging evidence: the heartbeat of professional traders in financial markets changes only with volatility, and not with, e.g., price drift, let alone its effect on awareness. Potentially fruitful starting points include failure anxiety and error awareness, which may be associated more readily. Recent findings point to a link between mis-assessment of signal-to-noise ratio and anxiety (see the article by Ullsperger et al. in this issue), and studies of error awareness have much in common with those of uncertainty, such as the dissociation between error anticipation and error encoding (see the article by M. Paulus and M. Stein in this issue). In the latter context, it is interesting to note that the sub-regional specialization between expectation of potential error (superior anterior insula) and post-response error recording (inferior anterior insula) bears resemblance with of the same specialization found in risk anticipation (superior) and risk prediction error (inferior) (see Fig. 3b). Likewise, the study of awareness of rare errors and the role of locus coeruleus parallels the development of neurobiological models of unexpected uncertainty ( $\mathrm{Yu}$ and Dayan 2003).

It is plausible to expect that uncertainty assessment is a crucial modulator of awareness. Organisms, whose environment changes too often for their innate reactions to always be well adapted require an alertness system that is triggered by uncertainty and that captures their bodily state (emotions) and mental state (feelings) sufficiently strongly so that learning ensues. Uncertainty carries a strong motivational component, inducing organisms to explore when gains are expected, and provoking defensive actions in harmful situations. The resulting actions can be expected to be more effective if they involve careful thought and planning, which requires awareness.

A theory that integrates feelings and uncertainty, in which anterior insula plays a crucial role has recently been advanced (Singer et al. 2009). In it, predictions and realizations of bodily states are combined with predictions and realizations of uncertainty, to generate an integrated feeling state that is shaped by individual (subjective) risk preferences. Here, we propose to add awareness into the picture.

Recently, financial decision making has been linked to awareness. Persaud et al. (2007) showed that betting success is an effective tool to measure awareness. The difference between performance in a physical exercise and success when betting on this performance appears to reflect subliminal processing. This finding not only constitutes a first step towards formalization of the link between 
awareness and risk assessment, but also shows that financial decision making requires awareness. As such, financial decision making may become a crucial gauge with which to measure awareness.

The link between uncertainty and bodily states and the role of insula is easier to grasp. For instance, equally intense pain is perceived as more painful if it is less predictable, and the encoding of pain anticipation in anterior insula reflects the uncertainty of the pain stimulus rather than the expectation (Carlsson et al. 2006). Similarly, anxiety (neuroticism) is correlated with uncertaintyinduced insula brain activation (Paulus et al. 2003). Still, there is a dearth of studies that simultaneously manipulate uncertainty and bodily signals. Does heartbeat really increase with variance as (Lo and Repin 2002) suggests? What about skin conductance and other psychophysiological indicators? Do frontal lesion patients in the Iowa Gambling Task make disadvantageous decisions because they have no emotional anticipation of risk, and therefore, are not aware of the risks involved? etc.

Finally, because of the ubiquitous nature of uncertainty in the environment, it would be interesting to explore whether mal-adaptive behavior such as compulsive gambling or addiction, as well as mental illnesses such as anxiety disorder or schizophrenia are associated with problems in risk learning, and to what extent anterior insula is implicated. With respect to borderline personality disorder, evidence has recently emerged of abnormally low insula activation following rejection in the well-known trust game that can be interpreted as a lack of awareness that forecasts of trustee behavior were wrong. The absence of subsequent behavioral adjustment is consistent with this lack of error encoding, as we discussed before (King-Casas et al. 2008).

Acknowledgments Supported by Grants from the US National Science Foundation (SES-0527491) and the Swiss Finance Institute. The manuscript benefited from discussions with Kerstin Preuschoff and Tania Singer, and comments from two referees and the editor, although the usual disclaimer applies.

\section{References}

Bechara A, Damasio H, Tranel D, Damasio AR (1997) Deciding advantageously before knowing the advantageous strategy. Science 275:1293-1295

Behrens TE, Woolrich MW, Walton ME, Rushworth MF (2007) Learning the value of information in an uncertain world. Nat Neurosci 10:1214-1221

Bekaert G, Harvey CR, $\mathrm{Ng} \mathrm{A} \mathrm{(2005)} \mathrm{Market} \mathrm{integration} \mathrm{and}$ contagion. J Business 78:39-69

Berger JO (1985) Statistical decision theory and Bayesian analysis. Springer, New York

Berns GS, Capra M, Chappelow J, Moore S, Noussair C (2008) Nonlinear neurobiological probability weighting functions for aversive outcomes. NeuroImage 39:2047-2057
Bossaerts P, Preuschoff K (2009) Q-Learning the learning rate. In: Society for neuroeconomics annual meetings. Evanston

Brass M, Haggard P (2007) To do or not to do: the neural signature of self-control. J Neurosci 27:9141-9145

Bunzeck N, Dayan P, Dolan RJ, Duzel E (2010) A common mechanism for adaptive scaling of reward and novelty. Hum Brain Mapp. doi:10.1002/hbm.20939

Carlsson K, Andersson J, Petrovic P, Petersson KM, Hman A, Ingvar M (2006) Predictability modulates the affective and sensory-discriminative neural processing of pain. NeuroImage 32:1804-1814

Christopoulos GI, Tobler PN, Bossaerts P, Dolan RJ, Schultz W (2009) Neural correlates of value, risk, and risk aversion contributing to decision making under risk. J Neurosci 29:12574-12583

Craig AD (2002) How do you feel? Interoception: the sense of the physiological condition of the body. Nat Rev Neurosci 3:655666

Craig AD (2009) How do you feel—now? The anterior insula and human awareness. Nat Rev Neurosci 10:59-70

d'Acremont M, Gilli M, Bossaerts P (2009a) Predicting risk in a multiple stimulus-reward environment. In: Dreher JC, Tremblay L (eds) Handbook of reward and decision making. Academic Press, New York, pp 460-473

D’Acremont M, Lu Z-L, Li X, Van der Linden M, Bechara A (2009b) Neural correlates of risk prediction error during reinforcement learning in humans. NeuroImage 47:1929-1939

Delgado MR, Frank RH, Phelps EA (2005) Perceptions of moral character modulate the neural systems of reward during the trust game. Nature Neurosci 8:1611

Ellsberg D (1961) Risk, ambiguity, and the savage axioms. Q J Econ 75:643-669

Fiorillo CD, Tobler PN, Schultz W (2003) Discrete coding of reward probability and uncertainty by dopamine neurons. Science 299:1898-1902

Hsu M, Bhatt M, Adolphs R, Tranel D, Camerer CF (2005) Neural systems responding to degrees of uncertainty in human decision making. Science 310:1680-1683

Hsu M, Krajbich I, Zhao C, Camerer CF (2009) Neural response to reward anticipation under risk is nonlinear in probabilities. J Neurosci 29:2231-2237

Huettel S, Song A, McCarthy G (2005) Decisions under uncertainty: probabilistic context influences activation of prefrontal and parietal cortices. J Neurosci 25:3304-3311

King-Casas B, Sharp C, Lomax-Bream L, Lohrenz T, Fonagy P, Montague PR (2008) The rupture and repair of cooperation in borderline personality disorder. Science 321:806-810

Koch C, Preuschoff K (2007) Betting the house on consciousness. Nat Neurosci 10:140-141

Koenig S, Simmons RG (1994) Risk-sensitive planning with probabilistic decision graphs. In: Doyle J, Sandewall E, Torasso P (eds) “ $\{\mathrm{KR}\}$ '94: principles of knowledge representation and reasoning. Morgan Kaufmann, San Francisco, pp 363-373

Kroll Y, Levy H, Markowitz HM (1984) Mean-variance versus direct utility maximization. J Finance 39:47-61

Kuhnen CM, Knutson B (2005) The neural basis of financial risk taking. Neuron 47:763-770

Lo AW, Repin DV (2002) The psychophysiology of real-time financial risk processing. J Cogn Neurosci 14:323-339

Mihatsch O, Neuneier R (2002) Risk-sensitive reinforcement learning. Mach Learn 49:267-290

Nassar M, Wilson R, Heasly B, Gold J (2009) Updating beliefs in a changing world. In: Society for neuroscience annual meetings. Chicago

Nursimulu AD, Preuschoff K, Bossaerts P (2009) On the pertinence of risk learning for reward learning. In: Society for neuroeconomics annual meetings. Evanston, IL 
Paulus MP, Rogalsky C, Simmons A, Feinstein JS, Stein MB (2003) Increased activation in the right insula during risk-taking decision making is related to harm avoidance and neuroticism. NeuroImage 19:1439-1448

Persaud N, McLeod P, Cowey A (2007) Post-decision wagering objectively measures awareness. Nat Neurosci 10:257-261

Platel H, Price C, Baron JC, Wise R, Lambert J et al (1997) The structural components of music perception. A functional anatomical study. Brain 120:229-243

Pratt JW (1964) Risk aversion in the small and in the large. Econometrica 32:122-136

Preuschoff K, Bossaerts P (2007) Adding prediction risk to the theory of reward learning. Ann N Y Acad Sci 1104:135-146

Preuschoff K, Bossaerts P, Quartz SR (2006) Neural differentiation of expected reward and risk in human subcortical structures. Neuron 51:381-390

Preuschoff K, Quartz SR, Bossaerts P (2008) Human insula activation reflects risk prediction errors as well as risk. J Neurosci 28:27452752

Ramirez I, Sanchez MB, Fernandez MC, Lipp OV, Vila J (2005) Differentiation between protective reflexes: cardiac defense and startle. Psychophysiology 42:732-739
Rutishauser U, Mamelak AN, Schuman EM (2006) Single-trial learning of novel stimuli by individual neurons of the human hippocampus-amygdala complex. Neuron 49:805-813

Sanfey AG, Rilling JK, Aronson JA, Nystrom LE, Cohen JD (2003) The neural basis of economic decision-making in the ultimatum game. Science 300:1755-1758

Schervisch MJ (1997) Theory of statistics. Springer, New York

Seeley WW, Menon V, Schatzberg AF, Keller J, Glover GH et al (2007) Dissociable intrinsic connectivity networks for salience processing and executive control. J Neurosci 27:2349-2356

Singer T, Lamm C (2009) The social neuroscience of empathy. Ann N Y Acad Sci 1156:81-96

Singer T, Critchley HD, Preuschoff K (2009) A common role of insula in feelings, empathy and uncertainty. Trends Cogn Sci 13:334-340

Tobler PN, Fiorillo CD, Schultz W (2005) Adaptive coding of reward value by dopamine neurons. Science 307:1642-1645

Tom SM, Fox CR, Trepel C, Poldrack RA (2007) The neural basis of loss aversion in decision-making under risk. Science 315:515518

Yu AJ, Dayan P (2003) Expected and unexpected uncertainty: ACh and NE in the neocortex. Adv Neural Inform Process Syst 15 